\section{Gravidez na adolescência e outros fatores de risco para mortalidade fetal e infantil no Município do Rio de Janeiro, Brasil}

\author{
Teenage pregnancy and other risk factors for fetal \\ and infant mortality in the city of Rio de Janeiro, \\ Brazil
}

\author{
${ }^{1}$ Escola Nacional de Saúde \\ Pública Sergio Arouca, \\ Fundação Oswaldo Cruz, Rio \\ de Janeiro, Brasil. \\ Correspondência \\ E. F. V. Oliveira \\ Escola Nacional de Saúde \\ Pública Sergio Arouca, \\ Fundação Oswaldo Cruz. \\ Rua Leopoldo Bulhões 1480, \\ 8 o andar, Rio de Janeiro, $R J$ \\ 21041-210, Brasil. \\ efvoliveira@ensp.fiocruz.br
}

\begin{abstract}
This article analyzes teenage pregnancy and other risk factors for fetal and infant mortality in the city of Rio de Janeiro, Brazil. The study included a sample of births in Rio de Janeiro, and exposure variables were organized hierarchically. For fetal death, the results showed the protective effect of adequate prenatal care, while maternal morbidity increased the risk. For neonatal death, adequate prenatal care and female gender in the newborn were protective factors, while black or brown maternal skin color, history of stillbirth, maternal morbidity, and physical aggression during the index pregnancy increased the risk. Low birth weight and prematurity were corroborated as determinants of fetal and neonatal death. Risk of post-neonatal death was highest with increased parity, intra-gestational morbidity, and low birth weight. Teenage pregnancy itself was an independent factor for post-neonatal death. The findings reaffirm the relevance of social and health policies targeting adolescents, as well as improvements in prenatal care.
\end{abstract}

Pregnancy in Adolescence; Fetal Mortality; Infant Mortality; Prenatal Care
Elaine Fernandes Viellas de Oliveira 1 Silvana Granado Nogueira da Gama 1 Cosme Marcelo Furtado Passos da Silva ${ }^{1}$

\section{Introdução}

Nas duas últimas décadas, o Brasil vem experimentando uma mudança importante na condição de saúde da população infantil. Ainda que persistam as desigualdades regionais, houve uma redução nos óbitos em crianças menores de um ano de vida em todo o país, fato decorrente, sobretudo, da queda mais expressiva no componente pós-neonatal 1 .

Na cidade do Rio de Janeiro, entre 1996 e 2001, a taxa de mortalidade infantil (TMI) apresentou um comportamento de queda constante, com redução de 25,7\% no coeficiente. Entre 2002 e 2004, observou-se uma estabilidade, com a TMI em torno de 15 para cada mil que nasceram vivos, retomando a tendência de declínio em 2005 e 2006, quando a taxa chegou a 13,6 para cada mil nascidos vivos ${ }^{1}$.

Vários fatores, tradicionalmente conhecidos, podem determinar a ocorrência dos óbitos infantis como, por exemplo, a prematuridade, o baixo peso ao nascer, asfixia grave ao nascer (índice de Apgar entre 0-3 no 1o minuto), a gemelaridade, a alta paridade, o baixo nível de escolaridade materna, a baixa renda familiar e a idade materna avançada 2,3,4,5,6.

Além dos fatores referidos, outros vêm sendo apontados, em especial o risco social para a mortalidade infantil, e, dentre esses, o risco da gravidez mais precoce. Utilizando as bases LILACS e MEDLINE, dentre os estudos que investigam 
a relação entre a mortalidade fetal ou infantil e a gravidez na adolescência, na última década, a maioria apresenta resultados positivos para a associação. Os achados indicam que os filhos das adolescentes apresentam maior probabilidade de morte durante o primeiro ano de vida, comparados aos de mães com 20 anos e mais de idade. A magnitude da associação aponta riscos em torno de 1,20 a 4,00, oscilando conforme a faixa etária de comparação da idade materna e dos óbitos analisados 7,8,9,10,11,12,13,14.

No entanto, vale comentar que existem importantes fatores que podem estar confundindo as associações. Segundo César et al. 15, as diferenças encontradas na mortalidade de crianças filhas de mães adolescentes podem estar refletindo mais um "efeito-pobreza" do que propriamente um "efeito-idade", sendo também um indicativo da precariedade ou mesmo ausência de uma assistência pré-natal adequada.

Quanto aos estudos que encontram associação negativa, de acordo com Fonseca \& Coutinho 16 , pode ser que os resultados controversos e não significativos ocorram devido a diferenças regionais ou por questões metodológicas, como os diferentes pontos de corte, o instrumento de coleta utilizado e a forma de registro das variáveis nos bancos de dados, por exemplo. Também adicionam que o pequeno tamanho amostral de alguns estudos pode contribuir para a não associação.

Este estudo objetiva analisar os fatores de risco para mortalidade fetal e infantil no Município do Rio de Janeiro, com atenção especial ao papel da gravidez na adolescência nessa relação.

\section{Métodos}

O trabalho faz parte do Estudo da Morbi-mortalidade e da Atenção Peri e Neonatal no Município do Rio de Janeiro, desenvolvido com base em uma amostra de puérperas que se hospitalizaram em maternidades do município por ocasião do parto, entre julho de 1999 e março de 2001.

Os estabelecimentos de saúde foram estratificados e agrupados em três tipos de estratos: (1) municipais e federais; (2) estaduais, militares e filantrópicos conveniados com o Sistema Único de Saúde (SUS); (3) privados. Foi selecionada uma amostra de, aproximadamente, $10 \%$ de parturientes do número previsto de partos hospitalares para cada estrato. Por questões logísticas, foram excluídos os estabelecimentos com menos de 200 partos por ano, correspondente a apenas $3,7 \%$ do total de estabelecimentos.

O tamanho da amostra em cada estrato foi estabelecido com o objetivo de comparar propor- ções em amostras iguais no nível de significância de $5 \%$ e detectar diferenças de, pelo menos, $3 \%$ com poder do teste de $90 \%$, baseando-se na proporção de baixo peso ao nascer $(<2.500 \mathrm{~g})$.

Ao final da pesquisa, foram realizadas 10.072 entrevistas. Ao todo, foram selecionadas 47 instituições: 12 compuseram o primeiro estrato amostral, correspondendo a $34,8 \%$ dos partos; 10 , o segundo estrato, correspondendo a $34,4 \%$ dos partos; e 25, o terceiro, com 30,8\% dos partos. As perdas contabilizaram $4,5 \%$ do total de partos ocorridos, sendo os principais motivos a alta precoce da mãe ou sua recusa em participar da pesquisa.

Os dados foram coletados dos prontuários maternos e dos recém-nascidos, além de entrevistas com as mães no pós-parto imediato, por acadêmicos bolsistas de Enfermagem e Medicina devidamente treinados e supervisionados pelos coordenadores. As informações foram obtidas mediante assinatura, pela mãe ou seu responsável, quando necessário, de um termo de consentimento livre e esclarecido. Ao final da pesquisa, foram realizadas 10.072 entrevistas. Considerando os partos múltiplos, o total de nascimentos correspondeu a 10.186.

Para a inclusão do desfecho "óbito infantil", foi realizado um linkage entre dois bancos de dados: 10.186 nascidos vivos dessa amostra e 5.946 óbitos infantis ocorridos no Município do Rio de Janeiro, no período de junho de 1999 a março de 2002. Os dados sobre mortalidade infantil foram coletados a partir do Sistema de Informações sobre Mortalidade (SIM), fornecido pela Coordenação de Epidemiologia da Secretaria Municipal de Saúde do Rio de Janeiro. O linkage dos dados foi feito pelo método do relacionamento probabilístico de registros, cujo procedimento completo está descrito por Pereira et al. 17 .

Considerando os 103 óbitos fetais, 80 óbitos neonatais e 14 pós-neonatais que foram identificados no trabalho de campo e o total de óbitos do processo de linkage (28 óbitos neonatais e 42 óbitos pós-neonatais), foram identificados 267 óbitos na amostra de nascimentos.

Para o presente estudo, foram excluídos os filhos de mães com 35 anos e mais para analisar adequadamente o efeito da maternidade na adolescência, obtendo assim uma amostra com 9.041 nascimentos e 228 óbitos, sendo 91 fetais, 90 neonatais e 47 pós-neonatais.

As definições de nascido vivo, óbito fetal, óbito neonatal e pós-neonatal foram as descritas na 10a revisão da Classificação Internacional de Doenças (CID-10) 6 .

Foi empregado, para análise, o modelo hierarquizado descrito por Fuchs et al. 18. A variável resposta foi o óbito, analisado por meio de três 
modelos distintos: um para óbito fetal, um para óbito neonatal e outro para óbito pós-neonatal. Cada variável dependente foi categorizada em dois níveis, apresentando distribuição de Bernoulli, na qual o tipo de óbito estudado recebeu valor 1 , e o não-óbito recebeu valor 0 .

A seleção das variáveis independentes foi realizada considerando-se os referenciais teóricos sobre os determinantes da mortalidade fetal e infantil, que são apresentados na Figura 1. Os fatores foram organizados por nível de proximidade com o desfecho, em grupos de variáveis: distais (características socioeconômicas e demográficas); intermediário I (características maternas); intermediário II (assistência pré-natal e parto) e proximais (características e condições de saúde do recém-nascido).

No nível distal, para representar características socioeconômicas e demográficas, foram escolhidas: a cor da pele, nível de escolaridade e o trabalho materno. A cor da pele da mãe foi referida pela puérpera e, na análise hierarquizada, foi agrupada em duas categorias branca e amarela; preta e parda. A escolaridade foi analisada em anos de estudo (séries completas). O trabalho materno remunerado foi categorizado em 1 (sim) e 0 (não).

Para avaliar as características maternas, no nível intermediário I, foram consideradas: a idade materna, a história reprodutiva, a morbidade materna, o comportamento materno, as características psicossocias e a exposição à agressão física. A idade materna foi medida em anos completos. Em relação à história reprodutiva, foram considerados o número de gestações, de natimortos e de prematuros anteriores. Também foi verificado o histórico de aborto. Para verificar a presença de morbidade, foram selecionadas as patologias:

\begin{tabular}{l} 
Características socioeconômicas e demográficas \\
$\begin{array}{l}\text { Nível Distal } \\
\text { Características socioeconômicas: escolaridade materna, trabalho materno } \\
\text { Características demográficas: raça/cor }\end{array}$ \\
\begin{tabular}{|l} 
Nível Intermediário I \\
Características maternas
\end{tabular} \\
$\begin{array}{l}\text { Idade } \\
\text { História reprodutiva: paridade, história de natimorto, história de prematuridade, história de aborto } \\
\text { Morbidade materna: doenças hipertensivas, diabetes, sífilis, infecção urinária, infecção pelo HIV, anemia } \\
\text { Comportamento materno: fumo, ingestão de bebida alcóolica, uso de drogas } \\
\text { Características psicossociais: situação conjugal, pai do bebê no domicílio, apoio do pai do bebê, } \\
\text { satisfação do pai do bebê, desejo materno quanto à gravidez, companheiro acompanhando ida à maternidade } \\
\text { Exposição à violência física }\end{array}$ \\
\hline
\end{tabular}

\begin{tabular}{|l|} 
Nível Intermediário II \\
Assistência pré-natal e parto
\end{tabular}

\begin{tabular}{l|}
\multicolumn{1}{c|}{\begin{tabular}{c|} 
Nível Proximal \\
Características e condições de saúde do recém-nascido
\end{tabular}} \\
Sexo \\
Condições de saúde do recém-nascido: idade gestacional, peso ao nascer, gemelar
\end{tabular}

\section{Desfecho}

Óbito fetal, neonatal e pós-neonatal 
doenças hipertensivas, diabetes, sífilis, infecção urinária, infecção pelo HIV e anemia. No entanto, a análise cuidadosa das variáveis apontou que apenas a doença hipertensiva e o diabetes poderiam permanecer no modelo, tendo em vista a robustez dos dados. Dessa forma, apenas essas patologias foram selecionadas, fossem preexistentes ou gestacionais. O comportamento materno abordou informações sobre o hábito de fumar, a ingestão de bebidas alcoólicas e o uso de drogas durante a gestação. Para avaliar as características psicossocias, foram investigados a situação conjugal, a satisfação e o apoio do pai do bebê em relação à gestação atual; a presença do pai do bebê no domicílio e a presença do companheiro no momento da admissão na maternidade. Em relação à ocorrência de violência doméstica, foi verificada a exposição à agressão física durante a gravidez. A pergunta foi entremeada no questionário utilizado, para as demais variáveis, junto às informações da gestação atual.

No nível intermediário II, a assistência prénatal foi analisada a partir de um escore de adequação de utilização do pré-natal, adaptado por Leal et al. 19, em 2004, - o índice de Kotelchuck modificado (Tabela 1). Ele avalia o número de consultas de pré-natal baseado no mês de seu início e na proporção de consultas observadas sobre o número de consultas esperadas, de acor- do com a idade gestacional no nascimento. Para análise hierarquizada, duas categorias foram constituídas, uma unindo os grupos 1 e 2 (não fez e inadequado), e outra, os grupos 3, 4 e 5 (intermediário, adequado e mais que adequado). A assistência ao parto foi analisada por meio do tipo de parto realizado (cesárea ou normal) e da peregrinação pelas maternidades em busca de internação para o parto.

Por fim, no nível proximal, as características e condições de saúde do recém-nascido foram representadas pelo sexo do concepto, o tipo de gestação (única ou gemelar), o peso ao nascer $(<2.500 \mathrm{~g} \mathrm{e} \geq 2.500 \mathrm{~g})$ e a idade gestacional $(<37 \mathrm{e}$ $\geq 37$ semanas). Para a idade gestacional, foi priorizada a informação pela data da última mestruação, e, quando não se dispunha dessa, foi usada a ultra-sonagrafia. Na ausência de ambas, utilizouse informação fornecida pela mãe.

A regressão logística foi realizada usando os programs SPSS versão 13.0 (SPSS Inc., Chicago, Estados Unidos) e o R versão 2.5.1 (The R Foundation for Statistical Computing, Viena, Áustria; http://www.r-project.org). Na construção do modelo de forma hierarquizada, as variáveis do primeiro nível (distal), significantes na análise univariada (teste de Wald ao nível de 25\%), foram selecionadas para compor o modelo multivariado. As variáveis significativas nesse modelo

Tabela 1

Classificação da adequação do pré-natal, segundo Leal et al. ${ }^{19}$.

Índice de Kotelchuck original

Não tem.

Gestantes que iniciaram o pré-natal após o 4ㅇ mês de gestação e fizeram menos de $50 \%$ das consultas esperadas (inadequado).

Gestantes que iniciaram os cuidados pré-natais antes ou durante o 4o mês e fizeram 50 a $79 \%$ das consultas (intermediário).

Gestantes que iniciaram os cuidados pré-natais antes ou durante o 4으 mês e fizeram 80 a 109\% das consultas (adequado).

Gestantes que tiveram o início do pré-natal antes ou durante o 4o mês e tiveram $110 \%$ de consultas ou mais em relação ao esperado para a idade gestacional (mais que adequado).
Índice de Kotelchuck modificado *

Não fez pré-natal.

Idem ao original; mais as mulheres que haviam iniciado o pré-natal após o 4 o mês e que fizeram um número de consultas maior do que $50 \%$ do esperado, bem como mulheres que tiveram um número de consultas abaixo de $50 \%$ do esperado, embora tenham iniciado o pré-natal até o 4o mês de gestação.

Idem ao original

Idem ao original

Idem ao original

* Escore proposto por Kotelchuck 41 e adaptado por Leal et al. 19. Avalia o número de consultas de pré-natal, baseado no mês de início do pré-natal e na proporção de consultas observadas sobre o número de consultas esperadas, de acordo com a idade gestacional no nascimento. 
multivariado, considerando 10\% de significância estatística, foram conservadas no modelo e entraram no ajuste do próximo bloco (intermediário I). O mesmo procedimento foi empregado até que as variáveis proximais fossem ajustadas para as variáveis distais e intermediárias. As variáveis selecionadas em um determinado nível permaneceram nos modelos subseqüentes e foram mantidas mesmo que a inclusão de variáveis hierarquicamente inferiores alterasse sua significância. Ao final, estimaram-se as odds ratio (OR) brutas e as OR ajustadas no acréscimo de cada etapa da regressão hierarquizada, com seus respectivos intervalos de $90 \%$ de confiança (IC90\%). A adoção dos valores 25\% e 10\% para significância estatística e do IC90\% se justificam pela baixa incidência da variável de desfecho na população estudada.

Utilizou-se a estatística deviance (função desvio) para avaliar o ajuste dos modelos. A contribuição das variáveis foi avaliada pela diferença entre as deviances de cada nível e pelo Critério de Informação de Akaike (AIC). A deviance foi avaliada pela distribuição qui-quadrado $\left(\chi^{2}\right) \mathrm{com}$ seus graus de liberdade. O AIC não tem distribuição associada, e quanto menor o valor, melhor o ajuste 20 .

Inúmeros modelos foram construídos, quer testando as variáveis na forma contínua ou categorizada, com o intuito de se obter estimativas mais precisas das OR ajustadas e um modelo estatístico mais parcimonioso. A qualidade do ajuste final do modelo foi avaliada pelos gráficos de resíduos padronizados de Pearson, resíduos padronizados deviance, envelope, leverage e distância de Cook.

Este estudo foi submetido e aprovado pelo Comitê de Ética para Pesquisa da Escola Nacional de Saúde Pública Sergio Arouca, Fundação Oswaldo Cruz. A confidencialidade da informação quanto ao conteúdo e à identificação da puérpera, assim como o anonimato por ocasião da publicação dos resultados serão assegurados.

\section{Resultados}

Das 9.041 puérperas que permaneceram no estudo, $22 \%$ eram adolescentes, com idade entre 12 e 19 anos. Dentro desse grupo, a proporção de mães que tiveram filhos antes dos 16 anos foi de $2,5 \%$. A média de idade foi de 17,4 anos para as mães adolescentes e 26,3 para puérperas entre 20 e 34 anos.

Em relação aos óbitos, foram identificadas diferenças importantes: as mães das crianças que vieram a falecer apresentaram maior freqüência de cor da pele preta ou parda, de episódios de agressão física na gestação, de morbidades prégestacionais e durante a gestação, assim como precisaram peregrinar mais à procura de local para a internação para o parto. Tais puérperas receberam menor apoio do pai do bebê e realizaram pior pré-natal que aquelas cujos bebês não chegaram ao óbito (Tabela 2).

Quanto à escolaridade e à idade da mãe, as médias foram mais baixas dentre as puérperas que tiveram, como desfecho, os óbitos dos seus bebês. No tocante à história reprodutiva, as médias também foram piores nesse grupo.

Os óbitos mostraram-se fortemente associados com as condições de saúde do recém-nascido, sendo a melhor utilização da assistência prénatal o fator que mais se destacou na proteção dos óbitos fetais e infantis.

A Tabela 3 apresenta os resultados obtidos para o modelo final. Na identificação dos fatores de risco distais, a cor da pele preta e parda da mãe mostrou associação significativa com o óbito neonatal, e o seu nível de escolaridade, com os óbitos fetais e pós-neonatais, porém uma associação estatisticamente não significativa. $\mathrm{O}$ risco de morte para os bebês no período neonatal foi 1,80 vez maior do que para aquelas com cor da pele branca ou amarela. A escolaridade, por sua vez, conferiu proteção para o óbito $(\mathrm{OR}=0,97 \mathrm{e}$ 0,94 fetal e pós-neonatal, respectivamente), na medida em que aumentavam os anos de estudo da mãe. No entanto, seu efeito foi mediado pelas demais variáveis após a inclusão de níveis hierarquicamente mais próximos ao desfecho.

Quanto às variáveis do nível intermediário I, cabe destacar os diferentes efeitos da idade da mãe sobre os óbitos. Não foi encontrada associação entre os óbitos fetais e a idade da puérpera. Já para os óbitos neonatais e pós-neonatais, conferiu proteção conforme o seu aumento (OR = 0,96 e 0,90), ou seja, quanto mais jovens as mães, maior o risco para óbito no primeiro ano de vida dos seus filhos. No entanto, a associação com os óbitos no período neonatal deixou de ser estatisticamente significativa após o ajuste para as demais variáveis do modelo.

O número de gestações anteriores mostrou associação apenas com o óbito pós-neonatal, indicando que, quanto maior esse número, maior o risco de morte para o bebê $(\mathrm{OR}=1,32)$. Do mesmo modo, a história de filho natimorto se associou ao óbito neonatal $(\mathrm{OR}=1,93)$. O efeito da natimortalidade anterior foi intermediado pelas demais variáveis do modelo em relação aos óbitos fetais, conferindo risco 1,66 vez maior quando comparado ao grupo sem histórico desse agravo. A prematuridade também apresentou efeito indireto sobre os óbitos, aumentando o risco de mortalidade, no período pós-neonatal, em $75 \%$. 
Tabela 2

Análise univariada de fatores de risco para mortalidade fetal, neonatal e pós-neonatal, respectivos odds ratio (OR) bruta e intervalo de $90 \%$ de confiança (IC90\%).

\begin{tabular}{|c|c|c|c|c|c|}
\hline \multirow[t]{2}{*}{ Variáveis } & \multicolumn{2}{|c|}{ Óbito fetal e infantil } & \multirow{2}{*}{$\begin{array}{l}\text { Óbito fetal } \\
\text { OR bruta } \\
\text { (IC90\%) }\end{array}$} & \multirow{2}{*}{$\begin{array}{l}\text { Óbito neonatal } \\
\text { OR bruta } \\
\text { (IC90\%) }\end{array}$} & \multirow{2}{*}{$\begin{array}{l}\text { Óbito pós- } \\
\text { neonatal } \\
\text { OR bruta } \\
\text { (IC90\%) }\end{array}$} \\
\hline & $\begin{array}{c}\text { Sim } \\
(N=228) \\
n(\%)\end{array}$ & $\begin{array}{c}\text { Não } \\
(\mathrm{N}=8.813) \\
n(\%)\end{array}$ & & & \\
\hline \multicolumn{6}{|l|}{ Cor da pele } \\
\hline Branca/Amarela & $96(42,5)$ & $4.578(52,3)$ & - & 1,00 & - \\
\hline Preta/Parda & $130(57,5)$ & $4.181(47,7)$ & - & $2,38(1,54-3,69)$ & - \\
\hline Escolaridade (anos) & - & - & $0,88(0,81-0,96)$ & - & $0,84(0,77-0,92)$ \\
\hline Idade materna (anos) & - & - & - & $0,94(0,90-0,97)$ & $0,92(0,88-0,97)$ \\
\hline Número de gravidez anterior & - & - & - & - & $1,27(1,06-1,53)$ \\
\hline Número de nascido morto anterior & - & - & $2,40(1,48-3,90)$ & $2,49(1,70-3,66)$ & - \\
\hline Número de prematuro anterior & - & - & - & - & $2,14(1,45-3,17)$ \\
\hline \multicolumn{6}{|l|}{ Morbidade da mãe } \\
\hline Hipertensão arterial sistêmica e diabetes preexistente & $19(8,5)$ & $563(6,4)$ & $3,03(1,52-6,01)$ & - & - \\
\hline Hipertensão arterial sistêmica e diabetes na gestação & $24(10,7)$ & $451(5,1)$ & - & $2,26(1,16-4,38)$ & $2,92(1,32-6,46)$ \\
\hline Agressão física na gestação & $15(6,6)$ & $303(3,4)$ & - & $4,31(2,30-8,10)$ & - \\
\hline Apoio do pai do bebê & $191(84,5)$ & $7.849(89,3)$ & - & - & $0,37(0,19-0,71)$ \\
\hline \multicolumn{6}{|l|}{ Índice de Kotelchuck modificado } \\
\hline Grupo 1 e 2 & $136(64,2)$ & $2.502(30,0)$ & 1,00 & 1,00 & 1,00 \\
\hline Grupo 3 ao 5 & $76(35,8)$ & $5.835(70,0)$ & $0,17(0,10-0,30)$ & $0,24(0,16-0,37)$ & $0,29(0,16-0,50)$ \\
\hline Peregrinação para o parto & $80(35,2)$ & $2.174(24,7)$ & - & $2,78(1,84-4,19)$ & - \\
\hline \multicolumn{6}{|l|}{ Sexo } \\
\hline Masculino & $113(57,9)$ & $4.343(50,6)$ & - & 1,00 & - \\
\hline Feminino & $82(42,1)$ & $4.246(49,4)$ & - & $0,60(0,39-0,92)$ & - \\
\hline Idade gestacional $<37$ semanas & $151(71,6)$ & $983(11,7)$ & $\begin{array}{c}43,85 \\
(21,15-90,89)\end{array}$ & $\begin{array}{c}21,49 \\
(13,46-34,32)\end{array}$ & - \\
\hline Peso $<2.500 \mathrm{~g}$ & $124(68,1)$ & $729(8,5)$ & $\begin{array}{c}27,93 \\
(15,58-50,06)\end{array}$ & $\begin{array}{c}25,35 \\
(16,11-39,89)\end{array}$ & $9,53(5,52-16,43)$ \\
\hline
\end{tabular}

A presença de morbidade materna (hipertensão arterial e diabetes) associou-se aos três tipos de óbitos. Entretanto, o período de seu surgimento interferiu de forma diferenciada nos grupos. Morbidades pré-gestacionais incrementaram o risco de óbito no período fetal $(\mathrm{OR}=$ 2,43 ). Por outro lado, quando diagnosticadas durante a gravidez atual, associaram-se aos óbitos neonatais e pós-neonatais, conferindo ao bebê, respectivamente, um risco 2,08 e 3,01 vezes maior de óbito ao compará-los com aqueles cujas mães não tiveram esses agravos na gestação atual.

Os episódios de agressão física durante a gravidez apresentaram $\mathrm{OR}>2,5$ para óbito neonatal, entretanto não foi verificada sua associação com os óbitos fetais e pós-neonatais. Identificou-se um efeito protetor, indireto, para o óbito pósneonatal em filhos de mulheres que se sentiram apoiadas pelo pai do bebê durante a gestação, havendo redução de $48 \%$ do risco.

Quanto às variáveis do nível intermediário II, a adequação do pré-natal conferiu proteção para todos os modelos. Nas puérperas com pré-natal intermediário/adequado/mais que adequado houve redução de $49 \%$ do risco de óbito pósneonatal e neonatal. No grupo de óbitos fetais, a proteção foi ainda maior, $60 \%$.

A peregrinação da gestante em busca de internação para parto se mostrou associada ao óbito neonatal. Tais gestantes apresentaram risco quase duas vezes maior de óbito para os seus bebês do que as mães que conseguiram atendimento na primeira maternidade procurada. No 
Modelo hierarquizado final com odds ratio (OR) ajustada, seu respectivo intervalo de $90 \%$ de confiança (IC90\%), a estatística deviance e o Critério de Informação de Akaike (AIC).

\begin{tabular}{|c|c|c|c|c|c|c|}
\hline \multirow[t]{2}{*}{ Variáveis } & \multicolumn{2}{|c|}{ Óbito fetal } & \multicolumn{2}{|c|}{ Óbito neonatal } & \multicolumn{2}{|c|}{ Óbito pós-neonatal } \\
\hline & \multicolumn{2}{|c|}{ OR ajustada * (IC90\%) } & \multicolumn{2}{|c|}{ OR ajustada * (IC90\%) } & \multicolumn{2}{|c|}{ OR ajustada * (IC90\%) } \\
\hline \multicolumn{7}{|l|}{ Nível distal } \\
\hline \multicolumn{7}{|l|}{ Cor da pele } \\
\hline Branca/Amarela & \multicolumn{2}{|c|}{-} & \multicolumn{2}{|c|}{1,00} & \multicolumn{2}{|c|}{-} \\
\hline Preta/Parda & \multicolumn{2}{|c|}{-} & \multicolumn{2}{|c|}{$1,80 * *(1,14-2,85)$} & \multicolumn{2}{|c|}{-} \\
\hline Escolaridade (anos) & \multicolumn{2}{|c|}{$0,97(0,88-1,07)$} & \multicolumn{2}{|c|}{-} & \multicolumn{2}{|c|}{$0,94(0,84-1,05)$} \\
\hline \multicolumn{7}{|l|}{ Nível intermediário I } \\
\hline Idade materna (anos) & \multicolumn{2}{|c|}{-} & \multicolumn{2}{|c|}{$0,96(0,92-1,00)$} & \multicolumn{2}{|c|}{$0,90 * \star(0,84-0,97)$} \\
\hline Número de gravidez anterior & \multicolumn{2}{|c|}{-} & \multicolumn{2}{|c|}{-} & \multicolumn{2}{|c|}{$1,32 * \star(1,02-1,72)$} \\
\hline Número de nascido morto anterior & \multicolumn{2}{|c|}{$1,66(0,93-2,97)$} & \multicolumn{2}{|c|}{$1,93 * *(1,19-3,16)$} & \multicolumn{2}{|c|}{-} \\
\hline Número de prematuro anterior & \multicolumn{2}{|c|}{-} & \multicolumn{2}{|c|}{-} & $1,25(0$, & $76-2,07)$ \\
\hline Morbidade da mãe & & & & & & \\
\hline Hipertensão arterial sistêmica e diabetes preexistente & 2,43 ** ( & $, 17-5,05)$ & & & & - \\
\hline Hipertensão arterial sistêmica e diabetes na gestação & & & $2,08 * \star(1$ & $, 03-4,22)$ & $3,01 * *($ & $, 33-6,78)$ \\
\hline Agressão física na gestação & & & $2,63 * *(1$ & $, 30-5,35)$ & & - \\
\hline Apoio do pai do bebê & & & . & & $0,52(0$, & $26-1,02)$ \\
\hline Nível intermediário II & & & & & & \\
\hline Índice de Kotelchuck modificado & & & & & & \\
\hline Grupo 1 e 2 & & & 1, & & & 00 \\
\hline Grupo 3 ao 5 & $0,40 * *($ & ,21-0,74) & $0,61 * *(0$ & $, 38-0,97)$ & 0,61 (0, & $33-1,12)$ \\
\hline Peregrinação para o parto & & & $1,39(0$, & $39-2,17)$ & & - \\
\hline Nível proximal & & & & & & \\
\hline Sexo & & & & & & \\
\hline Masculino & & & 1, & & & - \\
\hline Feminino & & & $0,63 * *(0$ & $, 40-0,98)$ & & - \\
\hline Idade gestacional < 37semanas & 12,36 ** & $28-28,91)$ & $5,01 * \star(2$ & ,76-9,07) & & - \\
\hline Peso $<2.500 \mathrm{~g}$ & $5,72 * \star(2$ & $90-11,27)$ & $7,98 * \star(4$ & $53-14,04)$ & $7,65 * \star(4$ & ,34-13,46) \\
\hline Estatística deviance e AIC & Modelo nulo & Modelo final & Modelo nulo & Modelo final & Modelo nulo & Modelo final \\
\hline Estatística deviance & 512,59 & 360,51 & 741,10 & 539,75 & 470,19 & 405,67 \\
\hline $\mathrm{AIC}$ & 514,59 & 374,51 & 745,10 & 561,75 & 472,19 & 423,67 \\
\hline
\end{tabular}

* OR ajustada pelas variáveis socioeconômicas e demográficas, pelas variáveis que representam as características maternas, pelas variáveis de assistência pré-natal e parto e, por fim, as variáveis relacionadas ao recém-nascido;

** OR estatisticamente significativa $(p<0,10)$.

entanto, a inclusão das variáveis do nível proximal provocou mudanças no efeito da variável (OR = 1,39).

Na última etapa da análise hierarquizada, as variáveis proximais foram incorporadas àquelas selecionadas nas etapas anteriores. Quanto aos fatores de risco proximais, o sexo feminino conferiu proteção de 37\% para o óbito neonatal. Verificou-se que, quanto maior o peso ao nascimento, menor a ocorrência de óbitos. A análise do peso categorizado mostrou que o risco de óbito fetal é aumentado em 5,72 vezes, e o risco de óbito neonatal e pós-neonatal, em quase oito vezes quando o peso do feto ou do recém-nascido é inferior a $2.500 \mathrm{~g}$. A idade gestacional na forma contínua apresentou a mesma tendência do peso, porém associando-se aos óbitos fetais e neonatais. $\mathrm{O}$ risco de óbito fetal foi 12 vezes maior, e o de óbito neonatal, cinco vezes maior entre os prematuros.

Ao final, foram encontradas OR significativas para: morbidade materna preexistente (OR = 2,43; IC90\%: 1,17-5,05), adequação do pré-natal $(\mathrm{OR}=0,40$; IC90\%: 0,21-0,74), idade gestacional 
< 37 semanas (OR = 12,36; IC90\%: 5,28-28,91) e peso ao nascimento $<2.500 \mathrm{~g}(\mathrm{OR}=5,72$; $\mathrm{IC} 90 \%$ : 2,90-11,27) para os óbitos fetais.

A cor da pele da mãe preta ou parda $(\mathrm{OR}=$ 1,80; IC90\%: 1,14-2,85), história de nascido morto $(\mathrm{OR}=1,93$; IC90\%: 1,19-3,16), presença de morbidade (OR = 2,08; IC90\%: 1,03-4,22) e de agressão física na gestação (OR = 2,63; IC90\%: 1,30-5,35), adequação do pré-natal (OR = 0,61; IC90\%: 0,380,97 ), sexo feminino (OR $=0,63$; IC90\%: 0,40$0,98)$, idade gestacional $<37$ semanas $(\mathrm{OR}=5,01$; IC90\%: $2,76-9,07)$ e peso $<2.500 \mathrm{~g}(\mathrm{OR}=7,98$; IC90\%: 4,53-14,04) para os óbitos neonatais.

A menor idade materna $(\mathrm{OR}=0,90$; $\mathrm{IC} 90 \%$ : 0,84-0,97), o número de gestações anteriores $(\mathrm{OR}=1,32$; IC90\%: 1,02-1,72), a presença de morbidade materna na gestação $(\mathrm{OR}=3,01$; IC90\%: 1,33-6,78) e a idade gestacional < 37semanas (OR = 7,65; IC90\%: 4,34-13,46) para os óbitos pós-neonatais.

Após o ajuste, mesmo as demais variáveis dos níveis anteriores perdendo sua significância estatística, permaneceram nos modelos para representar os fatores de risco para os óbitos fetais e infantis no Município do Rio de Janeiro. Observando os valores obtidos pelo AIC nos modelos ajustados, nota-se que o modelo final explica melhor a variabilidade dos óbitos devido à redução mais marcada no valor das estatísticas deviance e menor valor do AIC, indicando um melhor ajuste quando comparado aos outros modelos (Tabela 3).

A Tabela 4 expõe os resultados brutos e ajustados do efeito da idade materna sobre os óbitos neonatais e pós-neonatais. Observa-se que o efeito da idade da mãe em relação aos óbitos no período neonatal é intermediado pelas demais variáveis do modelo. No entanto, exerce um efeito direto sobre a mortalidade pós-neonatal, mesmo após a inclusão de todos os níveis hierárquicos do modelo, com uma proteção de $10 \%$, indicando que, quanto maior a idade da mãe, menor é o risco de óbito da criança.

Vale ressaltar que muitas variáveis foram excluídas do modelo por perderem significância estatística com a inserção das variáveis dos níveis seguintes (teste de Wald a 25\%), a saber: trabalho materno; história de aborto; fumo, ingestão de bebida alcoólica ou uso de drogas durante a gestação; situação conjugal; viver com o pai do bebê; satisfação do pai do bebê; desejo materno quanto à atual gravidez; presença do companheiro na maternidade; tipo de parto; gemelaridade.

\section{Discussão}

Os resultados obtidos reafirmam, no Município do Rio de Janeiro, a contribuição de fatores socioeconômicos, assistenciais e psicossociais, das características maternas e da criança na determinação dos óbitos fetais e infantis.

As desigualdades raciais tornam-se expressão de disparidades sociais, sendo fatores condicionantes da maior dificuldade de acesso aos serviços e cuidados de saúde. O efeito protetor em relação ao óbito neonatal para os filhos de mulheres brancas é fato documentado na literatura, ainda quando controlado pelas condições socioeconômicas 21,22 .

A associação da idade materna com a mortalidade se deu de forma diferenciada em cada componente do óbito infantil e não foi significativa em relação à natimortalidade. No entanto, verificou-se uma tendência maior de óbitos no primeiro ano de vida à medida que diminuía a idade materna, apresentando um efeito direto sobre os óbitos pós-neonatais e um efeito indireto, intermediado por outras variáveis, sobre os neonatais.

Esses achados vão ao encontro de resultados de outras investigações as quais identificam maiores efeitos adversos no primeiro ano de vida em filhos de adolescentes 23,24. Em estudo realizado no período de 1990 a 1999, com mães adolescentes chilenas, foi encontrado risco 2,39 vezes maior para mortalidade infantil entre mães menores de 15 anos quando comparadas às adolescentes entre 15 e 19 anos 14 .

No modelo proposto, a idade materna foi considerada no nível intermediário por poder, de certo modo, sofrer influência das condições socioeconômicas (nível distal), não devendo ser entendida como característica puramente biológica. É possível que as maiores taxas de mortalidade encontradas nos filhos de adolescentes estejam refletindo mais as diferenças socioeconômicas e demográficas das famílias, do que propriamente um efeito da idade, o que já vem sendo defendido por outros autores 15,25.

Por outro lado, a maior associação com os óbitos pós-neonatais - reconhecidamente mais relacionados a fatores ambientais - permite supor que parte do efeito da idade precoce na mortalidade pós-neonatal também possa estar associado à maior dificuldade da adolescente em estabelecer ligações afetivas com seu filho ${ }^{2}$. O fraco vínculo mãe/filho, somado à imaturidade $\mathrm{e}$ à baixa auto-estima da adolescente, pode resultar em desvantagens no cuidado e no desenvolvimento da criança 25 . No entanto, os motivos não estão totalmente claros, e não se pode afastar a presença de confundimento residual. A cor da 
Efeito da idade da mãe sobre os óbitos neonatais e pós-neonatais.

\begin{tabular}{lccc}
\hline & OR bruta (IC90\%) & OR ajustada * (IC90\%) & OR ajustada ** (IC90\%) OR ajustada *** (IC90\%) \\
\hline Óbito neonatal & $0,94 \#(0,90-0,97)$ & $0,94 \#(0,90-0,98)$ & $0,96(0,92-1,00)$ \\
Óbito pós-neonatal & $0,92 \#(0,88-0,97)$ & $0,89 \#(0,83-0,96)$ & $0,91 \#(0,84-0,97)$
\end{tabular}

* OR ajustada para os fatores distais e do próprio nível;

** OR ajustada para os fatores distais, intermediários I e II;

*** OR ajustada para os fatores distais, intermediários e proximais;

\# OR estatisticamente significativa $(p<0,10)$.

pele e a escolaridade da puérpera, por exemplo, são variáveis potencialmente enviesadas pela presença de um confundimento residual do nível socioeconômico, contribuindo para a diferença encontrada 26 .

É importante ressaltar que, considerando uma proporção de $22 \%$ para gravidez na adolescência, as puérperas com idade inferior a 16 anos, embora em menor proporção, devem apresentar riscos mais elevados. O fato de ficar grávida tão cedo deveria servir para futuros cuidados de prevenção. Entretanto, estudos indicam que muitas dessas adolescentes repetem a gravidez no primeiro ano após o parto, comprometendo ainda mais as condições sociais e de saúde dessas jovens e dos seus filhos 27 .

Este estudo apontou também que tanto as morbidades maternas quanto a assistência prénatal inadequada estiveram associadas ao óbito fetal e ao neonatal. Em uma revisão da literatura sobre a mortalidade perinatal no Brasil, destacase que a maioria dos óbitos é considerada evitável, e as principais falhas apontadas se encontram na qualidade da assistência ao pré-natal, ao parto e à assistência neonatal ${ }^{28}$. Além das complicações na mulher, o diabetes gestacional e a pré-eclâmpsia são doenças relacionadas à máformação congênita, aos problemas respiratórios no concepto e ao crescimento intra-uterino restrito, por exemplo 29,30. Nesse sentido, destacase a importância do controle das morbidades maternas com a realização de um pré-natal de qualidade.

Quanto à associação encontrada entre a ocorrência de agressão física durante a gravidez e o óbito neonatal, Schoeps et al. 31, em 2007, em um estudo na região sul do Município de São Paulo, Brasil, também identificaram aumento em quase três vezes no risco de óbito no período neonatal na presença de maus-tratos durante a gravidez. Os achados das pesquisas sobre violência e saúde da criança sugerem que o risco de óbito geral- mente é maior no período neonatal, e que a violência doméstica faz parte da rede causal da mortalidade, sendo influenciada pelo tipo de união conjugal e com os nascimentos prematuros e de baixo peso ${ }^{32}$. No presente estudo, o tema não pôde ser amplamente explorado por não terem sido utilizadas escalas apropriadas para avaliar a violência doméstica. Entretanto, considerando a relevância do mesmo, aponta-se a necessidade de ampliar essa discussão nos serviços de saúde, uma vez que as redes de atenção ainda são precárias ou inexistentes.

No modelo proposto, a variável "receber o apoio do pai do bebê durante a gestação" mostrou efeito protetor para o óbito pós-neonatal, o que vai ao encontro de outros estudos que apontam a importância do apoio de membros particulares da rede social da mulher durante a gravidez. Sabroza et al. 33 mostraram, por exemplo, que o apoio do pai do recém-nascido exercia uma influência positiva na conduta da gestante, levando a uma maior aderência ao pré-natal, reduzindo resultados adversos da gravidez.

O efeito protetor sobre a mortalidade no período pós-neonatal, neste estudo, pode estar indicando que, quando o apoio do pai do bebê é prévio ao nascimento, esse deve estar se estendendo nos primeiros meses de vida, sendo benéfico em termos de melhor bem-estar físico e psicológico, reduzindo o risco da mortalidade. Viver com o pai da criança, perceber sua satisfação com a nova família e sentir-se apoiada por ele influencia significativamente na satisfação da gestante, principalmente da adolescente, em relação à sua vida e à da criança 34 .

A importância do pré-natal na prevenção de resultados negativos da gestação e no período neonatal é bastante estabelecida na literatura. Seu acompanhamento adequado permite identificar situações de risco precocemente, reduzindo a ocorrência de óbitos 25,35. Neste estudo, a adequação do pré-natal teve sua magnitude di- 
minuída quando incluídas as variáveis do nível proximal, chegando a perder significância estatística no modelo para óbitos pós-neonatais. $\mathrm{O}$ efeito benéfico do cuidado pré-natal perdeu sua significância devido à inclusão de possíveis mediadores como a idade gestacional e o peso ao nascer da criança.

A redução desse efeito protetor do pré-natal sobre a mortalidade no período pós-neonatatal é esperada na medida em que a idade da criança vai aumentando. Dessa forma, a atenção integral à saúde do menor de um ano passa a ser do profissional específico dessa área, com a adoção de medidas preconizadas capazes de promover a saúde e prevenir a mortalidade infantil no Município do Rio de Janeiro.

Merece destaque a dificuldade para obter internação no momento do parto, fator ainda relevante para a morbi-mortalidade materna e infantil 36. Leal et al. 37, analisando dados deste mesmo estudo, demonstraram que, nos hospitais vinculados ao SUS, a peregrinação foi duas vezes maior em comparação àquelas cujo parto foi realizado nas instituições privadas, sugerindo uma possível associação entre peregrinação e condições de vida desfavoráveis, que, por sua vez, repercutem na mortalidade dos recémnascidos.

Cabe destacar que, de acordo com Menezes et al. 38 , quanto mais nova a gestante, maior a dificuldade para encontrar um leito para realizar o parto. Mulheres com mais de 35 anos de idade têm $40 \%$ mais chance de não precisar peregrinar para ter o filho do que as adolescentes com menos de 17 anos.

Quanto aos fatores proximais, verificou-se associação estatisticamente significativa entre o sexo masculino e o óbito neonatal, fato confirmado por outros autores 3,39 . Um dos motivos do maior risco de óbito para o sexo masculino no período neonatal pode estar relacionado ao amadurecimento pulmonar mais precoce no sexo feminino 39 . No estudo de Duarte \& Mendonça ${ }^{3}$, a maior incidência de doença da membrana hialina foi a principal causa de morbi-mortalidade entre os meninos. Entretanto, as causas para essas diferenças ainda são pouco investigadas.
O baixo peso ao nascer e a prematuridade são reconhecidos como os principais preditores da mortalidade fetal e neonatal. Além disso, durante o primeiro ano de vida, o efeito do baixo peso ao nascer se estende para o domínio do crescimento e desenvolvimento infantis, tornando-se também relevante para os óbitos pós-neonatais 2,40 . O forte efeito dessas variáveis proximais nos desfechos reforça a importância da qualidade da assistência pré-natal e ao parto, bem como do incentivo ao aleitamento materno, triagem neonatal e outros cuidados preconizados para o primeiro ano de vida 25,36 .

Sabendo-se que as adolescentes representaram mais de um quarto das puérperas atendidas no SUS (estratos 1 e 2) 37 e que foi maior o risco de morbi-mortalidade infantil entre os seus filhos, no presente estudo, aponta-se o desafio de ampliar a atenção ao adolescente, enquanto uma questão de saúde pública. O enfrentamento dessa questão passa pela qualificação das políticas sociais e de saúde voltadas a esse grupo etário; pela redução das desigualdades socioeconômicas; melhoria da atenção pré-natal; entre outras.

A orientação sexual na rede de ensino não pode ser introduzida como mais um tópico do programa escolar, mas ser instituída partindo da educação continuada dos professores que se sintam aptos para tal. As questões acerca da sexualidade devem ser discutidas com os adolescentes de forma que se sintam seguros para fazer suas opções sexuais. Parece que derrubar esse tabu tem sido mais um desafio ainda não alcançado.

Destaca-se, como limitação do estudo, o número reduzido dos óbitos estudados, uma vez que a amostra foi calculada considerando a prevalência de baixo peso ao nascer, reduzindo o poder do teste. Entretanto, as análises isoladas de óbitos fetais, neonatais e pós-neonatais revelaram alguns aspectos distintos, justificando-se a importância desse estudo a partir de uma amostra representativa de nascimentos do Município do Rio de Janeiro, possibilitando um maior conhecimento e maior subsídio para intervenções, com informações tão qualificadas como essas. 


\section{Resumo}

O objetivo foi identificar o efeito da gravidez na adolescência e outros fatores de risco para a mortalidade fetal e infantil no Município do Rio de Janeiro, Brasil. O estudo foi desenvolvido em uma amostra de nascimentos do município, e as variáveis de exposição foram organizadas em níveis de hierarquia. Para óbito fetal, pré-natal adequado revelou efeito protetor, $e$ morbidade materna aumentou seu risco. No óbito neonatal, pré-natal adequado e recém-nascido do sexo feminino foram fatores de proteção. No entanto, cor da pele da mãe preta ou parda, história prévia de nascido morto, morbidade materna e agressão física na gestação elevaram o risco. O baixo peso e a prematuridade foram corroborados como determinantes do óbito fetal e neonatal. No óbito pós-neonatal, o risco foi mais elevado na presença de maior número de gestações anteriores, morbidade na gestação e o baixo peso ao nascer. A gravidez na adolescência surgiu com um efeito direto para o óbito pós-neonatal. Os achados reafirmam a relevância das políticas sociais e de saúde voltadas para os adolescentes e a melhoria da atenção pré-natal.

Gravidez na Adolescência; Mortalidade Fetal; Mortalidade Infantil; Cuidado Pré-Natal

\section{Referências}

1. Secretaria Municipal de Saúde do Rio de Janeiro. Mortalidade infantil. http://www.saude.rio.rj.gov. $\mathrm{br} / \mathrm{cgi} / \mathrm{public} /$ cgilua.exe/sys/reader/htm/prein dexview.htm?editionsectionid $=48 \&$ user=reader (accesado em 06/Jun/2009).

2. Baldin PE, Nogueira PC. Fatores de risco para mortalidade infantil pós-neonatal. Rev Paul Pediatr 2008; 26:156-60.

3. Duarte JLMB, Mendonça GAS. Fatores associados à morte neonatal em recém-nascidos de muito baixo peso em quatro maternidades no Município do Rio de Janeiro, Brasil. Cad Saúde Pública 2005; 21:181-91.

4. Machado CJ, Hill K. Determinantes da mortalidade neonatal e pós-neonatal no Município de São Paulo. Rev Bras Epidemiol 2003; 6:345-58.

5. Almeida MF, Novaes HMD, Alencar GP, Rodrigues LC. Mortalidade neonatal no Município de São Paulo: influência do peso ao nascer e de fatores sócio-demográficos e assistenciais. Rev Bras Epidemiol 2002; 5:93-107.

\section{Colaboradores}

E. F. V. Oliveira realizou o levantamento bibliográfico todas as etapas da análise e da elaboração do texto. S. G. N. Gama contribuiu na interpretação dos resultados, organização e redação do artigo. C. M. F. P. Silva colaborou em todas as etapas da análise dos dados e da redação final do artigo.
6. Organização Mundial da Saúde. Classificação estatística internacional de doenças e problemas relacionados à saúde, 10a revisão. v. 1. São Paulo: Centro Colaborador da OMS para a Classificação de Doenças em Português; 1995.

7. Menifield CE, Dawson J. Infant mortality in southern states: a bureaucratic nightmare. J Health Hum Serv Adm 2008; 31:385-402.

8. Chen XK, Wen SW, Fleming N, Yang Q, Walker MC. Increased risks of neonatal and postneonatal mortality associated with teenage pregnancy had different explanations. J Clin Epidemiol 2008; 61: 688-94.

9. Malamitsi-Puchner A, Boutsikou T. Adolescent pregnancy and perinatal outcome. Pediatr Endocrinol Rev 2006; 3 Suppl 1:170-1.

10. Rodríguez Nuñez AC, Hernández Cruz I. Factores que inciden en la mortalidad fetal tardía. $\mathrm{Rev} \mathrm{Cu}-$ bana Obstet Ginecol 2004; 30(2):0-0. 
11. Gilbert W, Jandial D, Field N, Bigelow P, Danielsen B. Birth outcomes in teenage pregnancies. J Matern Fetal Neonatal Med 2004; 16:265-70.

12. Alexander MR, Salihu HM, Rouse DJ. Survival of triplets who are born to teen mothers in the United States. Am J Obstet Gynecol 2004; 191:2097-102.

13. Simões VMF, Silva AAM, Bettiol H, Lamy-Filho F, Tonial SR, Mochel EG. Características da gravidez na adolescência em São Luís, Maranhão. Rev Saúde Pública 2003; 37:559-65.

14. Donoso SE, Becker VJ, Villarroel PL. Birth rates and reprodutive risk in adolescents in Chile, 1990-1999. Rev Panam Salud Pública 2003; 14:3-8.

15. César CC, Ribeiro PM, Abreu DMX. Efeito-idade ou efeito-pobreza? Mães adolescentes e mortalidade neonatal em Belo Horizonte. Rev Bras Estud Popul 2000; 17:177-96.

16. Fonseca SC, Coutinho ESF. Pesquisa sobre mortalidade perinatal no Brasil: revisão da metodologia e dos resultados. Cad Saúde Pública 2004; 20 Suppl 1:S7-19.

17. Pereira APE, Gama SGN, Leal MC. Mortalidade infantil em uma amostra de nascimentos do Município do Rio de Janeiro, 1999-2001: "linkage" com o Sistema de Informação de Mortalidade. Rev Bras Saúde Matern Infant 2007; 7:83-8.

18. Fuchs SC, Victora CG, Fachel J. Modelo hierarquizado: uma proposta de modelagem aplicada à investigação de fatores de risco para diarréia grave. Rev Saúde Pública 1996; 30:168-78.

19. Leal MC, Gama SGN, Netto Ratto KM, Cunha CB. Uso do índice de Kotelchuck modificado na avaliação da assistência pré-natal e sua relação com as características maternas e o peso do recém-nascido no Município do Rio de Janeiro. Cad Saúde Pública 2004; 20 Suppl 1:S63-72.

20. Cook R, Weisberg S. Applied regression including computing and graphics. New York: John Wiley; 1999.

21. Carvalho PI, Pereira PMH, Frias PG, Vidal SA, Figueiroa JN. Fatores de risco para mortalidade neonatal em coorte hospitalar de nascidos vivos. Epidemiol Serv Saúde 2007; 16:185-94.

22. Hessol NA, Fuentes-Afflick E. Ethnic differences in neonatal and postneonatal mortality. Pediatrics 2005; 115:44-51.

23. Chen X, Wen SW, Fleming N, Demissie K, Rhoads GG, Walker M. Teenage pregnancy and adverse birth outcomes: a large population based retrospective cohort study. Int J Epidemiol 2007; 36 368-73.

24. Gama SGN, Szwarcwald CL, Leal MC. Experiência de gravidez na adolescência, fatores associados e resultados perinatais entre puérperas de baixa renda. Cad Saúde Pública 2002; 18:153-61.

25. Vieira MLF, Bicalho GG, Silva JLCP, Barros Filho AA. Crescimento e desenvolvimento de filhos de mães adolescentes no primeiro ano de vida. Rev Paul Pediatr 2007; 25:343-8.

26. Kaufman JS, Cooper RS, McGee DL. Socioeconomic status and health in blacks and whites: the problem of residual confounding and resiliency of race. Epidemiology 1997; 8:621-8.

27. Rosa AJ, Reis AOA, Tanaka ACA. Gestações sucessivas na adolescência. Rev Bras Crescimento Desenvolv Hum 2007; 17:165-72.
28. Lansky S, França E, Leal MC. Mortalidade perinatal e evitabilidade: revisão da literatura. Rev Saúde Pública 2002; 36:759-72.

29. Villar J, Carroli G, Wojdyla D, Abalos E, Giordan D, Ba'aqeel H. Preeclampsia, gestational hypertension and intrauterine growth restriction, related or independent conditions? Am J Obstet Gynecol 2006; 194:921-31.

30. Nazer Herrera J, García Huidobro M, Cifuentes Ovalle L. Malformaciones congénitas en hijos de madres con diabetes gestacional. Rev Méd Chile 2005; 133:547-54.

31. Schoeps D, Almeida MF, Alecan GP, França Jr. I, Novaes HMD, Siqueira AAF, et al. Fatores de risco para mortalidade neonatal precoce. Rev. Saúde Pública 2007; 41:1013-22.

32. Lipsky S, Holt VL, Easterling TR, Critchlow CW. Impact of police-reported intimate partner violence during pregnancy on birth outcomes. Obstet Gynecol 2003; 102:557-64.

33. Sabroza AR, Leal MC, Gama SGN, Costa JV. Perfil sócio-demográfico e psicossocial de puérperas adolescentes no Município do Rio de Janeiro, Brasil - 1999-2001. Cad Saúde Pública 2004; 20 Suppl 1:S112-20.

34. Gama SGN, Szwarcwald CL, Sabroza AR, Branco VC, Leal MC. Fatores associados à assistência prénatal precária em uma amostra de puérperas adolescentes em maternidades do Município do Rio de Janeiro, 1999-2000. Cad Saúde Pública 2004; 20 Suppl 1:S101-11.

35. Victora CG, Barros FC. Infant mortality due to perinatal causes in Brazil: trends, regional patterns and possible interventions. São Paulo Med J 2001; 119:33-42.

36. Lansky S, França E, César CC, Monteiro Neto LC, Leal MC. Mortes perinatais e avaliação da assistência ao parto em maternidades do Sistema Único de Saúde em Belo Horizonte, Minas Gerais, Brasil, 1999. Cad Saúde Pública 2006; 22:117-30.

37. Leal MC, Gama SGN, Campos MR, Cavalini LT, Garbayo LS, Brasil CLP, et al. Fatores associados a morbi-mortalidade perinatal em uma amostra de maternidades públicas e privadas do Município do Rio de Janeiro, 1999-2001. Cad Saúde Pública 2004; 20 Suppl 1:S20-33.

38. Menezes DCS, Leite IC, Schramm JMA, Leal MC. Avaliação da peregrinação anteparto numa amostra de puérperas no Município do Rio de Janeiro, Brasil, 1999/2001. Cad. Saúde Pública 2006; 22:553-9.

39. Araújo BF, Bozzetti MC, Tanaka ACA. Mortalidade neonatal precoce no Município de Caxias do Sul: um estudo de coorte. J Pediatr (Rio J.) 2000; 76:200-6.

40. Passebon E, Bloch KV, Kale PL, Coeli CM. Associação entre peso ao nascer e mortalidade infantil no Município de Campos dos Goytacazes - RJ. Cad Saúde Coletiva 2006; 14:283-96.

41. Kotelchuck M. An evaluation of Kessner adequacy of prenatal care index and a proposed adequacy of prenatal care utilization index. Am J Public Health 1994; 84:1414-20.

Recebido em 13/Out/2009

Versão final reapresentada em 30/Dez/2009 Aprovado em 15/Jan/2010 